\title{
EFICIÊNCIA DE UTILIZAÇÃO DE NUTRIENTES PARA FORMAÇÃO DE RAÍZES FINAS E MÉDIAS EM POVOAMENTO DE TECA ${ }^{1}$
}

\author{
Maurel Behling², Júlio César Lima Neves³, Nairam Félix de Barros ${ }^{3}$, Caroline Barbizan Kishimoto ${ }^{4}$ e Luit
} Smit $^{5}$

\begin{abstract}
RESUMO - O conhecimento das características do sistema radicular auxilia na definição de práticas de preparo de solo e fertilização. O objetivo deste trabalho foi estimar a biomassa, o conteúdo de nutrientes em raízes, o comprimento e a área de raízes finas e médias e a eficiência de utilização de macronutrientes da teca (Tectona grandis) para formação de área radicular em diferentes profundidades do solo. A separação das raízes do solo foi feita estratificando-as em grossas ( $>5 \mathrm{~mm}$ ), médias $(2$ a $5 \mathrm{~mm}$ ) e finas $(<2 \mathrm{~mm})$, para determinação de $\mathrm{N}, \mathrm{P}, \mathrm{K}, \mathrm{Ca}, \mathrm{Mg}$ e S. As raízes médias e finas $( \pm 0,5 \mathrm{~g})$ foram digitalizadas e quantificadas por intermédio do programa QuantRoot ${ }^{\circledR}$, obtendo-se o diâmetro, a área e o comprimento. A biomassa total de raízes finas e médias foi de 1.335 e $1.258 \mathrm{~kg} \mathrm{ha}^{-1}$ respectivamente, e 56,2\% das raízes finas e 44,4\% das raízes médias concentram-se nos primeiros $20 \mathrm{~cm}$ do solo. O comprimento total de raízes finas foi de $8.237 \mathrm{~km} \mathrm{ha}^{-1}$, com $57,4 \%$ nos primeiros $20 \mathrm{~cm}$ de profundidade. A superfície total de raízes finas foi de $18.441 \mathrm{~m}^{2} \mathrm{ha}^{-1}$, com $57 \%$ dessa superfície nos primeiros $20 \mathrm{~cm}$ do solo. A quantidade total de nutrientes nas raízes, na camada de solo estudada (0-60 cm), em ordem decrescente, foi $\mathrm{K}>\mathrm{Ca}>\mathrm{N}>\mathrm{Mg}>\mathrm{P}>\mathrm{S}$. O fósforo e o enxofre são os nutrientes com maior eficiência de utilização para formação de área radicular em plantios de teca.
\end{abstract}

Palavras-chave: Tectona grandis; Área radicular específica; Nutrição florestal.

\section{NUTRIENT USE EFFICIENCY FOR THE FORMATION OF FINE AND MEDIUM ROOTS IN TEAK STANDS}

\begin{abstract}
Knowing root system characteristics aids in the definition of practices of soil preparation and fertilization. The objective of this work was to estimate the biomass, root nutrient content, fine and medium roots length and area and the macronutrient use efficiency for the teak (Tectona grandis) regarding the formation of roots area in different soil depths. The separation of roots from the soil was made stratifying them in thick ( $>5 \mathrm{~mm}$ ), medium (2 to $5 \mathrm{~mm}$ ) and fine roots ( $<2 \mathrm{~mm}$ ), for $\mathrm{N}, \mathrm{P}, \mathrm{K}, \mathrm{Ca}, \mathrm{Mg}$ and $\mathrm{S}$ determination. Medium and fine roots $( \pm 0.5 \mathrm{~g})$ were scanned and quantified by the QuantRoot ${ }^{\circledR}$ program, generating the diameter, area and length. The total fine and medium roots biomass was of 1.335 and $1.258 \mathrm{~kg} \mathrm{ha}^{-1}$, respectively, with $56.2 \%$ of the fine roots and $44.4 \%$ of the medium roots concentrated in the $0-20 \mathrm{~cm}$ layer. The total fine roots length was $8.237 \mathrm{~km} \mathrm{ha}^{-1}$ with $57.4 \%$ in the first $20 \mathrm{~cm}$ depth. The total fine roots surface was $18.441 \mathrm{~m} 2 \mathrm{ha}^{-1}$, with $57 \%$ of this surface in the first $20 \mathrm{~cm}$ of soil. The total amount of nutrients in the roots, in the studied soil layer $(0-60 \mathrm{~cm})$, in decreasing order, was $K>\mathrm{Ca}>N>\mathrm{Mg}>\mathrm{P}>\mathrm{S}$. Phosphorus and sulfur are the nutrients with higher use efficiency for roots area formation in teak stands.
\end{abstract}

Keywords: Tectona grandis; Specific root área; Forest nutrition.

\footnotetext{
${ }^{1}$ Recebido em 01.06.2010 aceito para publicação em 13.06.2014.

${ }^{2}$ Empresa Brasileira de Pesquisa Agropecuária, Embrapa Agrossilvipastoril, Brasil. E-mail: <maurel.behling@embrapa.br>.

${ }^{3}$ Departamento de Solos, Universidade Federal de Viçosa, Brasil. E-mail: <julio@solos.ufv.br> e <nfbarros@ufv.br>.

${ }^{4}$ Graduando em Agronomia, Universidade Federal de Viçosa, Brasil. E-mail: <carolkishi@hotmail.com>.

${ }^{5}$ Tectona Agroflorestal Ltda, Brazil. E-mail: <luitsmit@terra.com.br>.
} 


\section{INTRODUÇÃO}

A teca (Tectona grandis L.f), também conhecida por teak (Índia, Siam, Birmânia e Indonésia), teck (França), ojati (Java), may sak (Laos) e tiek (Alemanha), é uma espécie arbórea decídua de floresta tropical, pertencente à família Verbenaceae(SCHUHLI; PALUDZYSZYNFILHO, 2010). O interesse comercial pelo plantio da teca no Brasil começou no final da década de 1960 pela empresa Cáceres Florestal S.A., na região do Município de Cáceres, MT (TSUKAMOTO FILHO et al., 2003). Nos últimos anos, esse interesse se consolidou devido ao esgotamento das áreas de matas nativas e pressões por órgãos ambientais contra a exploração ilegal de madeira. A teca é alternativa para a produção de madeira nobre de alto valor agregado, em razão de suas qualidades silviculturais, industriais e comerciais, além de tronco retilíneo, facilidade de cultivo, pouco sujeita a pragas e doenças, madeira de excelente qualidade, valorização da beleza paisagística, resistência e durabilidade. No Estado de Mato Grosso, essa espécie é cultivada com sucesso, obtendo-se redução do ciclo para 25 a 30 anos, em comparação com os 80 anos na Ásia, região de origem (SCHUHLI; PALUDZYSZYN FILHO, 2010).

$\mathrm{O}$ aumento significativo dos plantios de teca se deve, em parte, às condições edafoclimáticas favoráveis da região. Apesar disso, é necessário conhecer as relações entre a água, o solo, a planta e a atmosfera para obtenção de produtividades economicamente rentáveis e que atendam às exigências de mercado. Nessas relações, é de grande importância a obtenção de informações complementares referentes ao desenvolvimento e distribuição do sistema radicular. A biomassa total de raízes não necessariamente é indicador da área total de absorção de água e nutrientes, e alterações na sua arquitetura podem ocorrer sem mudanças na biomassa total de raízes (HODGE, 2004; BØRJA et al., 2008; MAURICE et al., 2010; FINÉR et al., 2011).

É consenso que o fator que mais influencia a distribuição de suas raízes no solo é o material genético, embora a planta possa ser também influenciada por outros fatores relativos ao solo, como matéria orgânica, fertilidade, densidade, aeração, textura, temperatura e condições em que a espécie se desenvolve, a exemplo de competição e espaçamento entre árvores (GONÇALVES; MELLO, 2000; HODGE, 2004; MAURICE et al., 2010; FINÉR et al., 2011). Para a teca, pouco se tem estudado o seu sistema radicular, pois a grande maioria dos estudos realizados trata apenas do sistema radicular de sustentação, constituído por raízes grossas. Essa limitação está relacionada, principalmente, à complexidade de estudos dessa natureza, que envolvem grande demanda de tempo, mão de obra e custo elevado.

A eficiência de utilização de nutrientes (EUN), tanto para a formação de biomassa quanto para a formação de superfícies de aquisição de recursos (luz, água e nutrientes), pode variar em função das diferenças genotípicas que podem ocorrer e da interação genótipoambiente. Essas diferenças podem alterar a capacidade de absorção, transporte e utilização dos nutrientes pelas plantas (STEENBJERG; JAKOBSEN, 1963; MARSCHNER, 1995; CRAINE, 2006). Do ponto de vista nutricional, as espécies eficientes na utilização de nutrientes são aquelas capazes de sintetizar o máximo de biomassa e superfícies de aquisição de recursos por unidade de nutriente absorvido (BARROS et al., 1995; CALDEIRA et al., 2002; CRAINE, 2006).

Além do conteúdo de nutrientes, morfologia e distribuição da biomassa de raízes no perfil do solo, a eficiência de utilização dos nutrientes para formação de superfície radicular também contribui para estimar o aporte de nutrientes, em sistemas baseados no balanço nutricional entre a demanda pelas árvores para dada produtividade esperada e o que é suprido pelo solo, como é o caso do NUTRICALC (BARROS et al., 1995). O conhecimento das características do sistema radicular auxilia na definição de práticas de preparo de solo e fertilização (local e época de aplicação) e é fundamental para o entendimento e avaliação da magnitude de processos ecofisiológicos básicos, principalmente aqueles relacionados com a nutrição mineral e o balanço hídrico das árvores (GONÇALVES; MELLO, 2000).

Nesse contexto, o objetivo deste trabalho foi avaliar a biomassa, conteúdo de nutrientes de raízes finas, médias e grossas, comprimento, superfície e eficiência de utilização de nutrientes para formação de área radicular $\left(E_{U N} N_{A R}\right)$, raízes finas e médias, de um povoamento de teca (Tectona grandis) na região de Tangará da Serra, Estado de Mato Grosso.

\section{MATERIAL E MÉTODOS}

O trabalho foi realizado utilizando dados obtidos no Município de Tangará da Serra, Mato Grosso, em plantios da empresa Tectona Agroflorestal Ltda., localizada a $14^{\circ} 04^{\prime} 38^{\prime \prime}$ Sul e $57^{\circ} 03^{\prime} 45^{\prime \prime}$ 'Oeste, com 
altitude média de $387 \mathrm{~m}$, clima Ami (tropical, quente e subúmido), conforme a classificação de Köppen, com quatro meses secos (junho a setembro). A precipitação pluviométrica média anual é de $1.750 \mathrm{~mm}$, sendo janeiro, fevereiro e março os meses mais chuvosos. A temperatura média anual é de $24{ }^{\circ} \mathrm{C}$. A área apresenta topografia suave ondulada, e a classe de solo predominante é o Argissolo Vermelho eutrófico, com vegetação composta por mata de transição entre Floresta Amazônica e Cerrado.

O povoamento foi implantado nos meses de outubro a dezembro de 1998, no espaçamento 3 x 2,4 m. Na área foram distribuídas aleatoriamente três parcelas com 132 árvores cada uma (950,4 m²), com idade média de 7,5 anos, em 2006. Em cada parcela foram medidas as circunferências a 1,30 m de altura (CAP) de todas as árvores. A determinação da distribuição das raízes e de sua biomassa foi realizada na árvore com o diâmetro médio da parcela, utilizando-se como unidades amostrais cilindros de $30 \mathrm{~cm}$ de diâmetro, nas profundidades de 0-10, 10-20, 20-30, 30-40 e 40$60 \mathrm{~cm}$, distribuídos em coordenadas L (três buracos equidistantes do tronco da árvore na linha de plantio), E (quatro buracos equidistantes do tronco da árvore na entrelinha) e D (quatro buracos equidistantes do tronco da árvore na diagonal à linha e entrelinha de plantio) em um quarto da área útil das árvores amostradas. A parcela total de avaliação foi constituída de uma área de $1,5 \times 1,2 \mathrm{~m}(\mathrm{~L}=1,2 \mathrm{~m} ; \mathrm{E}=1,5 \mathrm{~m}$ e $\mathrm{D}=1,9 \mathrm{~m}$ ), num total de 11 unidades amostrais para cada profundidade, totalizando 55 unidades por árvore. Devido à textura muito argilosa do solo, foi feita a separação manual das raízes. Após a separação, as raízes foram estratificadas por classe de diâmetro em grossas (> $5 \mathrm{~mm}$ ), médias (2 a $5 \mathrm{~mm}$ ) e finas ( $<2 \mathrm{~mm}$ ), utilizando-se gabaritos com diâmetros-limite. Somente a biomassa de raízes vivas foi determinada e a raiz pivotante, desconsiderada nessa amostragem.

As amostras das diferentes classes de raízes foram secas em estufa $\left(75^{\circ} \mathrm{C}\right)$ até peso constante $( \pm 96 \mathrm{~h})$ e, depois, pesadas. As raízes, antes de serem submetidas à secagem, foram imersas inicialmente em uma solução de $\mathrm{HCl}$ 3,0\% por 30 seg e, em seguida, em água destilada por mais 30 seg (BATAGLIA et al., 1978).

Subamostras foram moídas e digeridas. No extrato nítrico-perclórico, determinaram-se P (colorimetria), K (fotometria de emissão de chama), S (turbidimetria de sulfatos) e Ca e Mg (espectrofotometria de absorção atômica), enquanto no extrato sulfúrico, o N (Kjedahl). Os conteúdos de N, P, K, Ca, Mg e S foram calculados com base nos teores e produção de biomassa seca.

Subamostras das raízes médias e finas ( \pm 0,5 g) foram digitalizadas, sendo as raízes médias e finas nas resoluções de 100 e 200 dpi, respectivamente, utilizando-se “scanner” HP Scanjet 8200. Para o processamento das imagens, utilizou-se o programa QuantRoot ${ }^{\circledR}$, convertendo os valores de pixel das imagens em valores de preto e branco (imagens binárias). Em seguida, usou-se o filtro de "Mediana”, para a redução de pixels isolados e, na sequência, a remoção manual de "ruídos” da imagem.

Com as imagens processadas, utilizou-se o programa QuantRoot ${ }^{\circledR}$, para obtenção do diâmetro, área e comprimento. Com o auxílio do processador de planilhas Microsoft ${ }^{\circledR}$ Office Excel 2003, complementou-se o cálculo do comprimento total e da superfície radicular. Após a digitalização, as raízes foram secas em estufa de circulação e renovação de ar a $75^{\circ} \mathrm{C}$ até peso constante e pesadas em balança com precisão de 0,001 g.

Os valores de área e comprimento de raízes obtidos foram relacionados com o volume de solo amostrado e com a biomassa seca de raízes, cujo resultado foi expresso em $\mathrm{g} \mathrm{dm}^{-3}, \mathrm{~m} \mathrm{dm}^{-3} \mathrm{e} \mathrm{m}^{2} \mathrm{dm}^{-3}$ de solo e $\mathrm{m}^{2} \mathrm{~kg}^{-1}$ de biomassa, extrapolando-se para o volume de solo e biomassa de 1 ha, correspondente a cada camada amostrada.

A eficiência de utilização dos nutrientes para formação de área radicular $\left(\mathrm{EUN}_{\mathrm{AR}}\right)$ foi obtida através da razão entre a área radicular específica $\left(\mathrm{ARE}=\mathrm{m}^{2}\right.$ $\mathrm{kg}^{-1}$ de biomassa seca de raiz) e o teor de nutrientes na raiz ( $\mathrm{g} \mathrm{kg}^{-1}$ ) (BARROS et al., 1986).

Os dados foram submetidos à análise estatística descritiva. A comparação das diferenças entre as classes de diâmetro das raízes sobre as variáveis estudadas em cada camada do solo foi feita através do erro-padrão da média.

\section{RESULTADOS}

A biomassa total de raízes foi de 21,9 $\mathrm{t} \mathrm{ha}^{-1}$. Desse total, 19,3 t ha-1 foram de raízes grossas, que variou na seguinte ordem: 6,81; 6,98; 3,16; 1,38; e 0,99 t ha-1 , nas profundidades de 0-10, 10-20, 20-30, 30-40 e 40-60 cm, respectivamente. Observou-se que $88 \%$ da biomassa de raízes grossas se encontrava na camada superficial do solo $(0-30 \mathrm{~cm})$ e $88 \%$ da biomassa de raízes finas e $77 \%$ das raízes médias estavam na camada de $0-40$ cm 
(Tabela 1). Assim como a biomassa, o comprimento de raízes finas ( $<2 \mathrm{~mm}$ ) também apresentou seus maiores valores nas camadas mais superficiais do solo. O comprimento médio de raízes finas até a profundidade de $60 \mathrm{~cm}$ do solo foi de $8.237 \mathrm{~km} \mathrm{ha}^{-1}$ (Tabela 1).

A superfície radicular apresentou o mesmo padrão de comportamento que a biomassa e o comprimento com um valor médio de $18.441 \mathrm{~m}^{2} \mathrm{ha}^{-1}$, e, em média, $72 \%$ da superfície radicular das raízes finas encontrava-se nos primeiros $30 \mathrm{~cm}$ de profundidade do solo. Nas raízes médias, $70 \%$ dos $4.813 \mathrm{~m}^{2} \mathrm{ha}^{-1}$ concentrava-se nos primeiros $30 \mathrm{~cm}$ do solo (Tabela 1 ).

A densidade de raízes finas foi maior que a densidade das raízes médias na camada superficial do solo (0$10 \mathrm{~cm}$ ), no entanto não diferiu nas demais camadas (Figura 1a). O maior valor de densidade de comprimento radicular das raízes finas ocorreu nos primeiros $10 \mathrm{~cm}$ do solo, com o valor médio de $3,1 \mathrm{~m} \mathrm{dm}^{-3}$, reduzindo em profundidade e alcançando um valor médio de $0,7 \mathrm{~m} \mathrm{dm}^{-3}$ na camada de 40 a $60 \mathrm{~cm}$. As raízes médias apresentaram variação em profundidade menor, com valores de $0,12 \mathrm{~m} \mathrm{dm}^{-3}$ na camada superficial, reduzindo para 0,05 $\mathrm{m} \mathrm{dm}^{-3}$ na camada mais profunda (Figura $1 \mathrm{~b}$ ).

A maior densidade de superfície radicular das raízes finas foi encontrada na camada superficial $(0$ a $10 \mathrm{~cm})$, com média de $6,8 \mathrm{~m}^{2} \mathrm{~m}^{-3}$ de solo, reduzindo gradativamente em profundidade e chegando à média de $1,5 \mathrm{~m}^{2} \mathrm{~m}^{-3}$ na camada de 40 a $60 \mathrm{~cm}$. Nas raízes médias, essa variação em profundidade foi menos pronunciada, variando de $1,1 \mathrm{~m}^{2} \mathrm{~m}^{-3}$ na camada de 0 a $10 \mathrm{~cm}$ a $0,4 \mathrm{~m}^{2}$ $\mathrm{m}^{-3}$ na camada de 40 a $60 \mathrm{~cm}$ (Figura $1 \mathrm{c}$ ).

A área radicular específica (ARE) foi, em média, de 13,86 e 4,35 $\mathrm{m}^{2} \mathrm{~kg}^{-1}$ nas raízes finas e médias, respectivamente. Portanto, por unidade de massa, a área de raízes finas foi aproximadamente 3,2 vezes maior que a de raízes médias. A variação em profundidade é pouca, ocorrendo diminuição da ARE com o aumento da profundidade. Os valores de ARE variaram de 14,71 m² $\mathrm{kg}^{-1}$ em superfície a $13,65 \mathrm{~m}^{2} \mathrm{~kg}^{-1}$ na camada mais profunda para raízes finas e de 4,86 $\mathrm{m}^{2} \mathrm{~kg}^{-1}$ em superfície a $3,79 \mathrm{~m}^{2} \mathrm{~kg}^{-1}$ na camada mais profunda para raízes médias (Figura 1d).

Os teores de N, K e S decresceram com o aumento da classe de diâmetro das raízes, ou seja, foram inversamente proporcionais à classe de diâmetro. No entanto, o contrário ocorreu com o $\mathrm{P}$, em que a maior concentração aconteceu nas raízes grossas ( $\geq 5 \mathrm{~mm}$ ). Já com relação ao Ca e ao Mg, a maior concentração se deu nas raízes da classe de diâmetro médio (2 a 5 mm). A concentração média, nas diferentes classes de raízes, de cada nutriente permite representá-los na seguinte escala decrescente: $\mathrm{K}>\mathrm{Ca}>\mathrm{N}>\mathrm{Mg}>\mathrm{S} \sim \mathrm{P}$ (Figura 2).

Tabela 1 - Biomassa seca, comprimento e superfície de raízes finas ( $<2 \mathrm{~mm}$ ) e médias ( 2 a $5 \mathrm{~mm}$ ), em diferentes profundidades do solo, para Tectona grandis, aos 7,5 anos de idade, no Município de Tangará da Serra, MT.

Table 1 - Dry biomass, length and surface of fine roots $(<2 \mathrm{~mm})$ and medium roots $(2-5 \mathrm{~mm})$, in different soil depths, to Tectona grandis, aged 7.5 years, in the municipality of Tangará da Serra, MT.

\begin{tabular}{|c|c|c|c|c|c|c|c|c|c|c|}
\hline \multirow{4}{*}{$\begin{array}{l}\text { Raízes } \\
\text { (f)Finas } \\
(<2 \mathrm{~mm})\end{array}$} & \multirow{3}{*}{$\begin{array}{c}\text { Prof. } \\
-\mathrm{cm}- \\
0-10\end{array}$} & \multicolumn{3}{|c|}{ Biomassa seca } & \multicolumn{3}{|c|}{ Comprimento } & \multicolumn{3}{|c|}{ Superfície } \\
\hline & & \multicolumn{2}{|c|}{$-\mathrm{kg} \mathrm{ha}^{-1}-\ldots$} & \multirow{2}{*}{$\frac{\%}{35,40^{2 \prime}}$} & \multicolumn{2}{|c|}{$---\mathrm{km} \mathrm{ha}^{-1}-\ldots$} & \multirow{2}{*}{$\frac{\%}{37,58}$} & \multicolumn{2}{|c|}{$-\mathrm{m}^{2} \mathrm{ha}^{-1}-\ldots$} & \multirow{2}{*}{$\frac{\%}{36,77}$} \\
\hline & & 472,68 & $( \pm 36,86)^{1 /}$ & & 3095,02 & $( \pm 247,32)$ & & 6780,97 & $( \pm 519,22)$ & \\
\hline & $10-20$ & 277,87 & $( \pm 21,89)$ & 20,81 & 1629,15 & & 19,78 & 3727,53 & & 20,21 \\
\hline & $20-30$ & 197,53 & $( \pm 11,44)$ & 14,79 & 1232,22 & & 14,96 & 2810,19 & & 15,24 \\
\hline & & 164,25 & $( \pm 10$ & & 5,66 & & & & & 12,03 \\
\hline & $40-60$ & 222,91 & $( \pm 28,61)$ & 16,69 & 1304,82 & $( \pm 178,37)$ & 15,84 & 2904,03 & $( \pm 350,83)$ & 15,75 \\
\hline & Total & 1335,23 & $( \pm 100,91)$ & $51,48^{\frac{3}{}}$ & 8236,87 & $( \pm 704$ & 94,04 & 18440,95 & $( \pm 1454$ & 79,30 \\
\hline (m)Médias & $0-10$ & 254,07 & $( \pm 46,89)$ & 20,19 & 117,26 & $( \pm 14$ & 22,45 & 1078,38 & $( \pm 145$ & 22,41 \\
\hline \multirow[t]{5}{*}{ (2 a $5 \mathrm{~mm})$} & $10-20$ & 304,15 & $( \pm 40,19)$ & 24, & 2,96 & & 25,46 & 1241,68 & & 25,80 \\
\hline & $20-30$ & 229,00 & $( \pm 24,90)$ & 18, & & & 19,04 & & & 18,84 \\
\hline & $30-40$ & 187,82 & $( \pm 33,79)$ & 14, & 77,39 & $( \pm 8,3$ & 14,82 & 702,61 & & 14,60 \\
\hline & $40-60$ & 283,36 & $( \pm 80,68)$ & 22,52 & 95,28 & $( \pm 17$, & 18,24 & 883,34 & $( \pm 174,30)$ & 18,35 \\
\hline & Total & 1258,39 & $( \pm 139,25)$ & 48,52 & 522,33 & $( \pm 41,82)$ & 5,96 & 4812,92 & $( \pm 401,93)$ & 20,70 \\
\hline Total $(\mathrm{f}+\mathrm{m})$ & & 2593,62 & $( \pm 119,75)$ & 100,00 & 8759,20 & $( \pm 684,85)$ & 100,00 & 23253,87 & $( \pm 1356,21)$ & 100,00 \\
\hline
\end{tabular}

1/.Valores entre parênteses referem-se ao erro-padrão da média;` Percentual de cada classe de raízes por camada do solo; e ${ }^{3 /}$ Percentual em relação à biomassa total de raízes finas e médias.

${ }^{1 /}$ The standard error of the mean; ${ }^{2 /}$ Percentage of each roots class per soil layer; $e^{3 /}$ Percentage relative to total biomass of fine and medium roots.

Revista Árvore, Viçosa-MG, v.38, n.5, p.837-846, 2014 


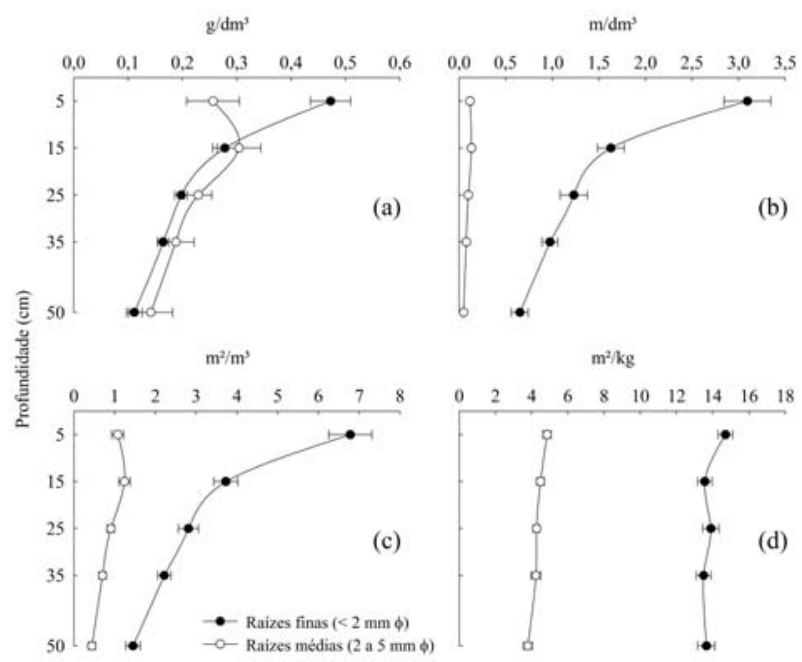

Figura 1 - Atributos morfométricos: (a) Densidade $\left(\mathrm{g} \mathrm{dm}^{-3}\right)$, (b) densidade de comprimento radicular ( $\left.\mathrm{m} \mathrm{dm}^{-3}\right)$, (c) densidade de superfície radicular $\left(\mathrm{m}^{2} \mathrm{~m}^{-3}\right)$ e (d) área radicular específica (ARE $=\mathrm{m}^{2} \mathrm{~kg}^{-1}$ de MS) de raízes finas e médias, em diferentes profundidades do solo, para Tectona grandis aos 7,5 anos de idade, no Munícipio de Tangará da Serra, MT. Barras horizontais em cada ponto representam o erro-padrão da média.

Figure 1 - Morphometric atributes: (a) Density $\left(\mathrm{g} \mathrm{dm}^{-3}\right)$. (b) root length density $\left(\mathrm{m} \mathrm{dm}^{-3}\right)$, (c) root surface density $\left(\mathrm{m}^{2} \mathrm{~m}^{-3}\right)$ and (d) specific root area (SRA $=\mathrm{m}^{2} \mathrm{~kg}^{-1}$ of DM) of thin and medium roots, in different soil depths, to Tectona grandis, aged 7.5 years, in the municipality of Tangará da Serra, MT. Horizontal bars in each point indicate standard error of the mean.
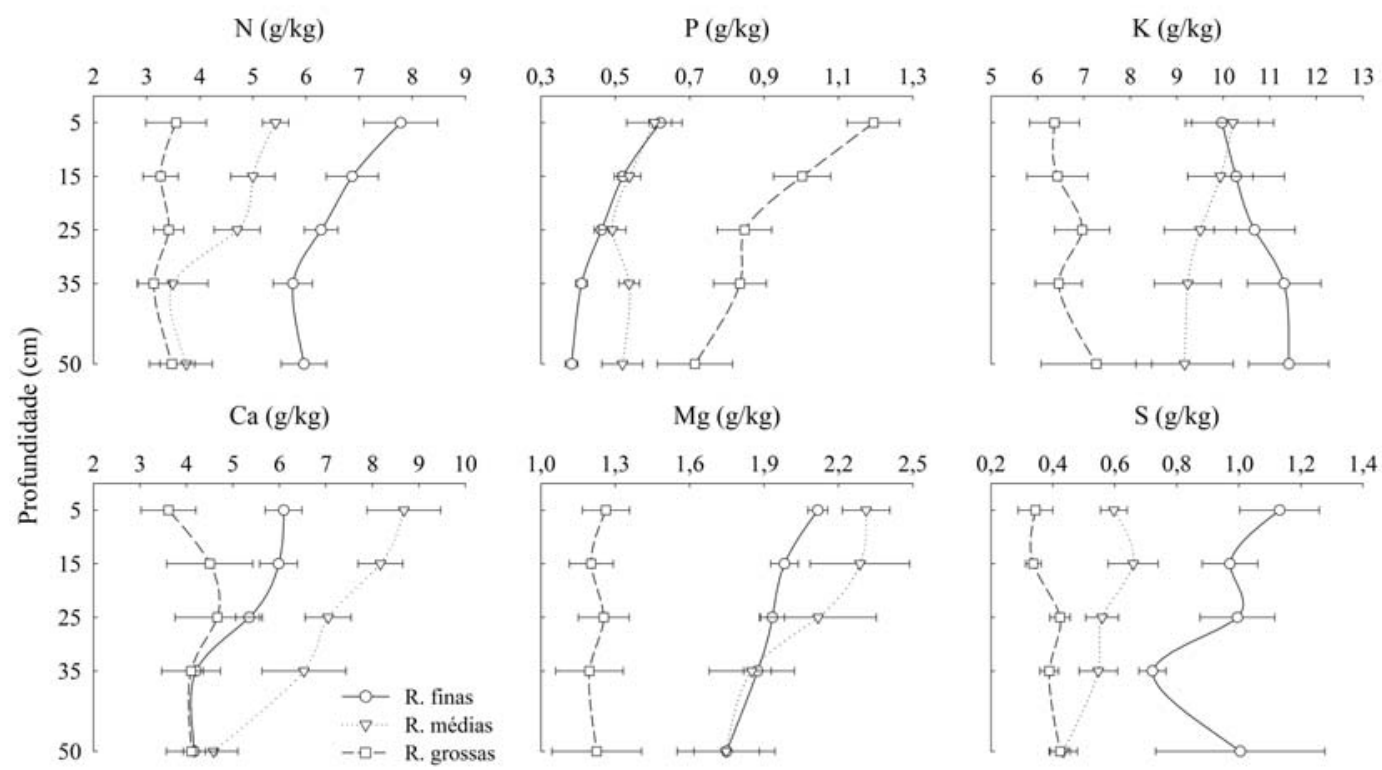

Figura 2 - Distribuição dos teores de macronutrientes de raízes grossas, médias e finas, em diferentes profundidades do solo, para Tectona grandis, aos 7,5 anos de idade, no Município de Tangará da Serra, MT. Barras horizontais em cada ponto representam o erro-padrão da média.

Figure 2 - Macronutrient content distribution of thick, medium and fine roots, in different soil depths, to Tectona grandis, aged 7.5 years, in the municipality of Tangará da Serra, MT. Horizontal bars in each point indicate standard error of the mean. 
A distribuição do conteúdo dos nutrientes no perfil do solo segue a tendência da biomassa, que é maior na superfície; por conseguinte, o conteúdo de nutrientes é alto, reduzindo em profundidade. Para raízes grossas e finas, bem como para o total de raízes (grossas + médias + finas), há, quase sempre, decréscimo dos conteúdos e, em geral, o máximo conteúdo ocorre na camada correspondente de 10 a $20 \mathrm{~cm}$ do perfil do solo. Observouse que a maior quantidade dos nutrientes $\mathrm{N}, \mathrm{P}, \mathrm{K}, \mathrm{Ca}$, Mg e S estão na camada superficial do solo de 0-20 $\mathrm{cm}$ de profundidade. A quantidade total de nutrientes na camada de solo estudada $(0-60 \mathrm{~cm})$, em ordem decrescente, foi $\mathrm{K}>\mathrm{Ca}>\mathrm{N}>\mathrm{Mg}>\mathrm{P}>\mathrm{S}$ (Figura 3).

Os maiores valores de eficiência de utilização dos nutrientes para formação de área radicular $\left(\mathrm{EUN}_{\mathrm{AR}}\right)$
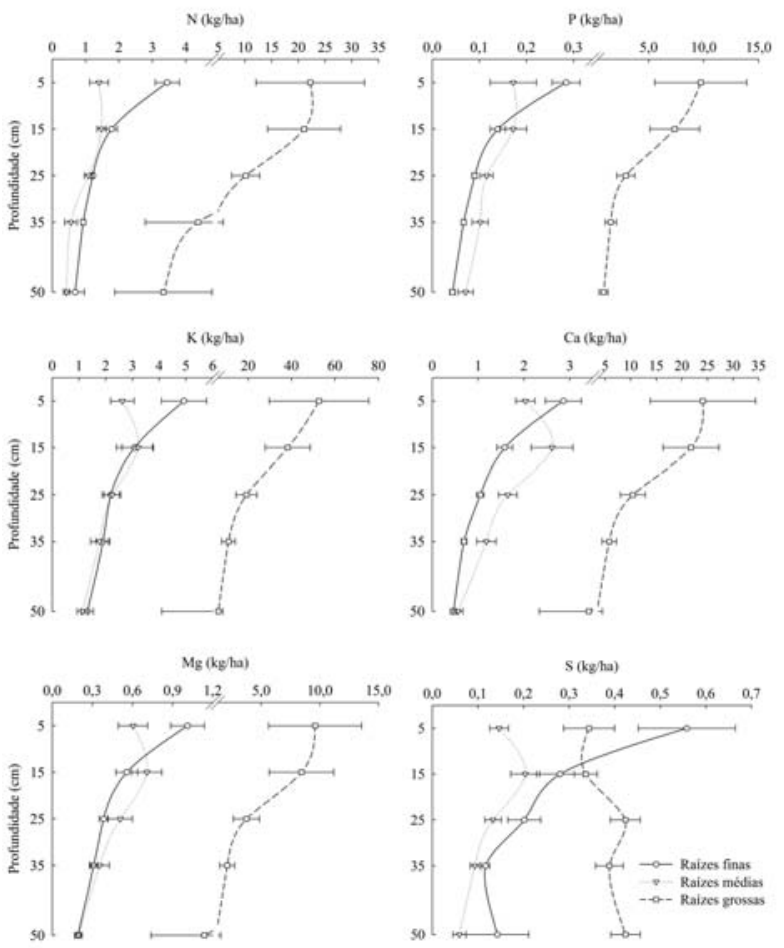

Figura 3 - Distribuição dos conteúdos de macronutrientes de raízes grossas, médias e finas, em diferentes profundidades do solo, para Tectona grandis, aos 7,5 anos de idade, no Município de Tangará da Serra, MT. Barras horizontais em cada ponto representam o erro-padrão da média.

Figure 3- Macronutrient content distribution of thick, medium and fine roots, in different soil depths, to Tectona grandis, aged 7.5 years, in the municipality of Tangará da Serra, MT. Horizontal bars in each point indicate standard error of the mean. foram encontrados para o P, seguido do S, Mg, Ca, $\mathrm{N}$ e K nas raízes finas. Isso indica que, entre os nutrientes avaliados, o P e o S foram os nutrientes utilizados com maior eficiência para produção de área radicular, enquanto o $\mathrm{N}$ e o $\mathrm{K}$ foram os nutrientes utilizados com menor eficiência pela planta. Observou-se para N, P, Ca, Mg e $\mathrm{S}$ aumento da $\mathrm{EUN}_{\mathrm{AR}}$ em profundidade das raízes finas, enquanto para o K a eficiência diminui. Quanto às raízes médias, a variação em profundidade da $\mathrm{EUN}_{\mathrm{AR}}$ foi menor (Figura 4).

\section{DISCUSSÃO}

Os resultados da biomassa total de raízes finas e médias, considerando todas as amostras coletadas na linha de plantio (L), entre linha de plantio (E) e diagonal
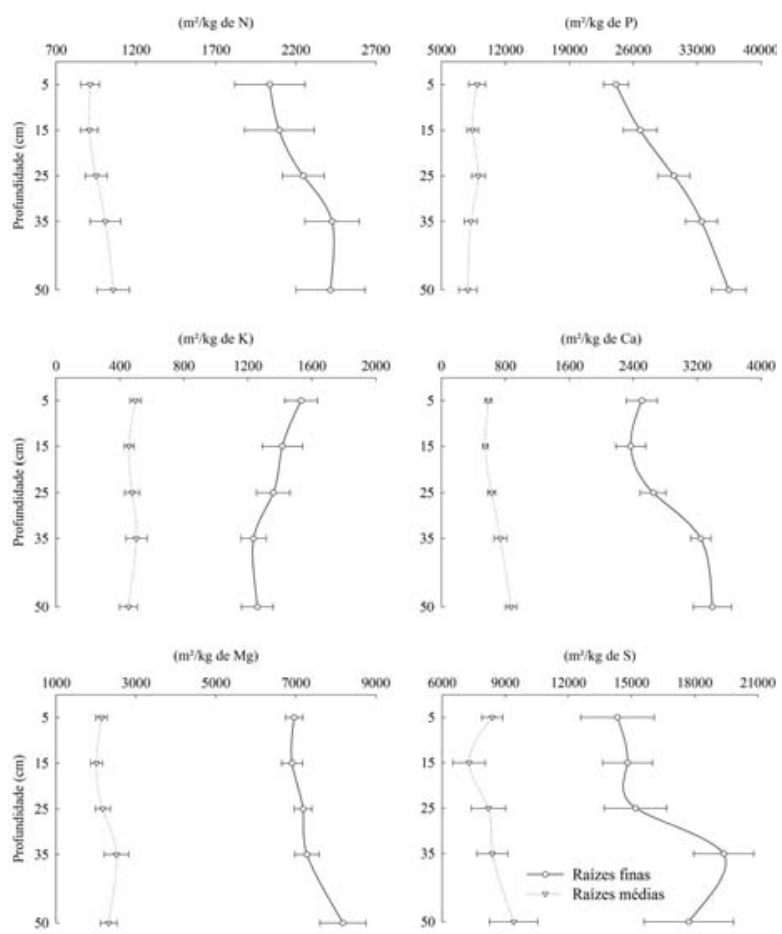

Figura 4 - Eficiência na utilização de macronutrientes para formação de superfícies radiculares $\left(\mathrm{EUN}_{\mathrm{AR}}\right)$ de raízes finas e médias, em diferentes profundidades do solo, para Tectona grandis, aos 7,5 anos de idade, Município de Tangará da Serra, MT. Barras horizontais em cada ponto representam o erro-padrão da média.

Figure 4-Macronutrients use efficiency for root surfaces formation $\left(N U E_{R A}\right)$ of fine and medium roots, in different soil depths, to Tectona grandis, aged 7.5 years, in the municipality of Tangará da Serra, MT. Horizontal bars in each point indicate standard error of the mean.

Revista Árvore, Viçosa-MG, v.38, n.5, p.837-846, 2014 
à linha e entre linhas de plantio (D) (Tabela 1), podem ser comparados com aqueles encontrados por Luy et al. (1997), que obtiveram em Eucalyptus grandis, aos

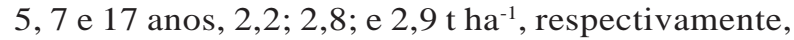
de raízes finas menores que $5 \mathrm{~mm}$ até a profundidade de $50 \mathrm{~cm}$, no solo. Esses valores são mais elevados que o valor médio de 1,34 t ha ${ }^{-1}$ obtido das raízes finas, classificadas com o diâmetro inferior a $2 \mathrm{~mm}$. Isso certamente influenciou tais resultados, independentemente da profundidade do solo. Quando se soma a biomassa de raízes finas $(<2 \mathrm{~mm})$ com a biomassa de raízes médias ( 2 a $5 \mathrm{~mm}$ ), esses valores se aproximam, obtendo-se uma média de 2,61 t ha-1 de raízes de teca $<5 \mathrm{~mm}$.

Schumacher (1995), com Eucalyptus bicostata, observou que a biomassa de raízes finas $(<2,0 \mathrm{~mm})$ diminuiu com o aumento da profundidade do solo, sendo o mesmo comportamento observado em teca (Tabela 1). A diferença na produção de biomassa radicular em relação a outros trabalhos ocorre em função da espécie, da idade, das condições edafoclimáticas e da profundidade amostrada, bem como da metodologia empregada (CRAINE, 2006; BØRJA et al., 2008). A comparação de resultados de biomassa radicular é difícil, pois os métodos usados para coletar as informações não são padronizados, além do fato de esse tipo de levantamento ser bastante trabalhoso (SCHUMACHER, 1995; MAJDI et al., 2005; CRAINE, 2006; BØRJA et al., 2008).

A concentração de $72 \%$ do comprimento das raízes finas em superfície, nos primeiros $30 \mathrm{~cm}$ de profundidade do solo (Tabela 1), está de acordo com dados observados por Mello et al. (1998), que encontraram, nos três materiais genéticos (Eucalyptus grandis, propagado por semente e dois híbridos do Eucalyptus grandis x Eucalyptus urophylla), 70\% das raízes ( $<3 \mathrm{~mm}$ ) nos primeiros 30 cm de profundidade do solo. Há correspondência entre a maior abundância de raízes nas camadas superficiais do perfil de solo e a maior disponibilidade de água e nutrientes (HODGE, 2004; MAURICE et al., 2010; FINÉR et al., 2011).

O comprimento de raiz varia em função da época de coleta e da espécie. Mello et al. (1998) constataram que no verão o comprimento de raiz de eucalipto (< 1,0 mm) variou de 5.500 a $25.200 \mathrm{~km} \mathrm{ha}^{-1}$ na serapilheira e de 91.400 a $53.100 \mathrm{~km} \mathrm{ha}^{-1}$ no perfil do solo, para o melhor clone e para o material propagado por semente, respectivamente. A extensão de raízes finas é característica intrínseca do genótipo, a qual é estreitamente relacionada com o seu comportamento nutricional, potencial produtivo e capacidade de adaptação às condições de estresse ambiental (CRAINE, 2006; OSTONEN et al., 2007; ZOBEL et al., 2007; MAURICE et al., 2010).

A produção de biomassa e o comprimento e superfície de raízes finas e médias por camada de solo diminuíram, em geral, com a profundidade. No entanto, a espessura das camadas de solo estudadas não é a mesma, exceto nas quatro primeiras camadas, sendo o padrão de produção de biomassa e do comprimento e superfície de raízes finas e médias ao longo do perfil de solo mais bem avaliado pelos valores de densidade radicular ( $\mathrm{g}$ $\left.\mathrm{dm}^{-3}\right)$, densidade de comprimento radicular $\left(\mathrm{m} \mathrm{dm}^{-3}\right)$, densidade de superfície radicular $\left(\mathrm{m}^{2} \mathrm{~m}^{-3}\right)$ e área radicular específica $\left(\mathrm{m}^{2} \mathrm{~kg}^{-1}\right)$ em relação ao volume de solo (Figura 1). Essas variáveis, indicadoras da plasticidade morfológica de raízes finas, refletem os mecanismos de resposta da planta à variação da disponibilidade de água e nutrientes (HODGE, 2004; CRAINE, 2006; BØRJA et al., 2008; FINÉR et al., 2011).

A densidade de raízes finas diminuiu com a profundidade do solo (Figura 1a), confirmando dados da literatura que revelam que a densidade de raízes é maior nas primeiras camadas do solo. Em geral, a biomassa de raízes finas diminui exponencialmente da superfície às camadas mais profundas do solo em diferentes biomas florestais, diferindo apenas na profundidade total de enraizamento (FINÉR et al., 2011). A densidade de raízes correlaciona-se com a taxa de crescimento de determinadas espécies. Estudo realizado com Eucalyptus grandis, com idade média de 5,6 anos, revelou que, quanto mais elevada era a taxa de crescimento das árvores, menor era a densidade de raízes finas (diâmetro $\leq 3,0 \mathrm{~mm}$ ) nas diferentes camadas do solo. As menores densidades de raízes foram encontradas nos sítios menos férteis (GONÇALVES; MELLO, 2000).

A densidade de raízes médias apresentou comportamento distinto ao das raízes finas (Figura 2a), com maior densidade na faixa de 10 a $20 \mathrm{~cm}$, acima da camada de maior compactação do solo. Isso evidencia a sensibilidade das raízes dessa classe de tamanho ao aumento de resistência do solo à penetração das raízes, fato observado por Neves (2000), em plantios clonais de eucalipto (Urograndis) no perfil de um Argissolo Amarelo, para essa mesma classe de tamanho

Revista Árvore, Viçosa-MG, v.38, n.5, p.837-846, 2014 
de raízes. Esse comportamento é também verificado em solos arenosos, sem compactação, em que o motivo para o maior adensamento das raízes médias, na camada de 10 a $20 \mathrm{~cm}$, seja o fato de as raízes terem pouco tempo para o pleno desenvolvimento na camada superficial do solo $(0-10 \mathrm{~cm})$. A camada superficial desse solo é um ambiente inóspito com efeitos adversos da radiação solar sobre a superfície exposta do solo, limitando o desenvolvimento de raízes (KING et al., 2002; MARTINS, 2004).

A distribuição da superfície específica de raízes $\left(\mathrm{m}^{2} \mathrm{~m}^{-3}\right)$ acompanha o padrão de distribuição da densidade de raízes $\left(\mathrm{g} \mathrm{dm}^{-3}\right)$. Comportamento semelhante foi observado por Berish e Ewel (1988) com raízes de espécies tropicais arbóreas da Costa Rica e por Schwengber et al. (1994), em raízes de seringueira (Hevea spp.).

A área radicular específica (ARE, $\mathrm{m}^{2} \mathrm{~kg}^{-1}$ ), observada neste estudo, dá ideia da eficiência com que o carbono (C) é usado para produzir superfície radicular. A maior área radicular específica (ARE), que corresponde à alta razão de área radicular (RAR em m² de área radicular por g de massa da planta), representa a maior taxa de crescimento e de renovação, refletindo na maior dinâmica das raízes finas em comparação com as médias. A rápida regeneração das raízes pode ser vantajosa em ambientes ricos em nutrientes, nos quais a eficiência na captura de recursos é maximizada pela redução da longevidade das raízes, aumentando a capacidade de absorção e reduzindo os custos de manutenção da planta (NORBY; JACKSON, 2000; CRAINE, 2006).

O decréscimo dos teores de macronutrientes, especialmente $\mathrm{N}$, K e S, com o aumento da classe de diâmetro das raízes, é reflexo da maior atividade metabólica nas raízes finas (MARSCHNER, 1995; GORDON; JACKSON, 2000; TAIZ; ZEIGER, 2004). Já os menores teores de macronutrientes das raízes nas camadas mais profundas de solo não podem ser atribuídos ao efeito de diluição, uma vez que a biomassa de raízes decresce com a profundidade (Tabela 1). Os decréscimos observados nos teores de nutrientes nas raízes finas podem indicar a existência de maior proporção de raízes jovens com maior atividade metabólica, nas camadas mais superficiais de solo, podendo também ser atribuídos à redução na disponibilidade dos nutrientes no solo em profundidade (NEVES, 2000).

Como o conteúdo de nutrientes é consequência da concentração e da produção de biomassa, a maior quantidade de nutrientes encontra-se alocada nas raízes grossas. Isso se deve à grande diferença em termos da quantidade de biomassa produzida, ou seja, essa classe representa $88 \%$ da biomassa total de raízes. A exceção é para o $S$, na camada de $0-10 \mathrm{~cm}$, cujo conteúdo foi maior nas raízes finas. Independentemente da profundidade, o K apresentou quantidade nas raízes superior a todos os demais nutrientes, sendo de $13,83 \mathrm{~kg} \mathrm{ha}^{-1}$ na camada superficial do solo $(0-20 \mathrm{~cm})$, considerando-se somente as raízes finas e médias. Quando se considerou a biomassa total de raízes, esse valor foi de $106 \mathrm{~kg} \mathrm{ha}^{-1}$.

Os resultados indicaram que, para a produção de área radicular, a teca demandou maior quantidade de $\mathrm{K}$ no solo, em relação à quantidade de $\mathrm{P}$, e maior quantidade de Ca no solo do que de $\mathrm{Mg}$. Devido às baixas concentrações e reservas de Ca na maioria dos solos usados para fins de reflorestamentos, as fertilizações devem prever, necessariamente, a aplicação de consideráveis quantidades de $\mathrm{Ca}$, junto com as fontes de N, P e K, ou por meio da calagem. No caso específico dos povoamentos de teca nas condições estudadas, plantados em solos com teores elevados de Ca, a sua reposição ao solo deve ser levada em consideração, visando à sustentabilidade do sistema de longo prazo, devido ao ciclo prolongado (25 anos) e à elevada exigência da espécie (ZHOU et al., 2010).

A maior eficiência no uso do P também foi observada em Mimosa caesalpiniifolia Benth por Moura et al. (2006), em Acacia mearnsii De Wild. por Caldeira et al. (2002) e em Eucalyptus saligna Smith e E. dunnii (Maid) por Schumacher (1995). Vitousek (1984) constatou que o P é o elemento que possui elevada eficiência de utilização nas florestas tropicais, principalmente em áreas onde ele se encontra em baixos teores. Já a elevada eficiência no uso do S pode ser atribuída aos elevados teores de argila e óxidos de ferro no solo, que resultaram numa alta capacidade de retenção de $\mathrm{SO}_{4}{ }^{-2}$, tornando a sua disponibilidade para a planta e movimentação no perfil mais lenta, comparativamente a solos com menor quantidade desses grupos funcionais (ALVAREZ V. et al., 2007).

Independentemente da classe de diâmetro das raízes, o K foi o nutriente que apresentou menor $\mathrm{EUN}_{\mathrm{AR}}$. Isso pode ter ocorrido em virtude da sua maior disponibilidade no solo (NEVES, 2000). O N também apresentou eficiência baixa, comparado aos demais nutrientes, o que, segundo Caldeira et al. (2002), se deveu aos altos teores nas folhas verdes e à retranslocação interna.Também, na 
presença de competição, há grande alocação de $\mathrm{N}$ para as raízes favorecida pela habilidade da planta em aumentar a densidade de raízes (CRAINE, 2006).

\section{CONCLUSÕES}

Nos primeiros $20 \mathrm{~cm}$ de solo, concentraram-se 56,2\% da biomassa seca, 57,4\% do comprimento total e 57\% da superfície das raízes finas de teca $(<2 \mathrm{~mm})$.

A densidade, o comprimento e a área radicular específica da teca diminuem com a profundidade do perfil, e os maiores valores concentram-se na camada de $0-20 \mathrm{~cm}$.

A ordem decrescente da quantidade total de nutrientes nas raízes, na camada de solo estudada $(0-60 \mathrm{~cm})$, foi $\mathrm{K}>\mathrm{Ca}>\mathrm{N}>\mathrm{Mg}>\mathrm{P}>\mathrm{S}$.

O fósforo e o enxofre são os nutrientes com maior eficiência de utilização, enquanto o nitrogênio e o potássio, os nutrientes com menor eficiência de utilização para formação de área radicular em plantios de teca, no Município de Tangará da Serra, Estado de Mato Grosso.

\section{REFERÊNCIAS}

ALVAREZ V., V. H. et al. Enxofre. In: NOVAIS, R. F. et al. (Ed.). Fertilidade do Solo. Viçosa, MG: Sociedade Brasileira de Ciência do Solo, 2007. v.1. p.595-644.

BARROS, N. F. et al. Classificação nutricional de sítios florestais - Descrição de uma metodologia. Revista Árvore, v.10, n.1, p.112-120, 1986.

BARROS, N. F. et al. NUTRICALC 2.0 - Sistema para calculo del balance nutricional y recomendación de fertilizantes para el cultivo de eucalipto. Bosque, n.16, p.129-131, 1995.

BatAglia, O. C.et al. Análise química de plantas. Campinas: Instituto Agronômico de Campinas, 1978. 31p.

BERISH, C. W.; EWEL, J. J. Root development in simple and complex tropical successional ecosystems. Plant and Soil, v.108, n.1, p.7384, 1988.

BØRJA, I. et al. Stand age and fine root biomass, distribution and morphology in a Norway spruce chronosequence in southeast Norway. Tree Physiology, v.28, p.773-784, 2008.
CALDEIRA, M. V. W.; RONDON NETO, R. M.; SCHUMACHER, M. V. Avaliação da eficiência nutricional de três procedências australianas de acácia-negra (Acacia mearnsii De Wild.).

Revista Árvore, v.26, n.5, p.615-620, 2002.

CRAINE, J. M. Competition for nutrients and optimal root allocation. Plant and Soil, v.285, p.171-185, 2006.

FINÉR, L. et al. Factors causing variation in fine root biomass in forest ecosystems. Forest Ecology and Management, v.261, p. 265-277, 2011.

GORDON, W. S.; JACKSON, R. B. Nutrient concentration in fine roots. Ecology, v.81, n.1, p.275-280, 2000.

GONÇALVES, J. L. M.; MELLO, S. L. M. O sistema radicular das árvores. In: GONÇALVES, J. L. M. (Ed.) Nutrição e fertilização de florestas. Piracicaba: IPEF, 2000. p.221-267.

HODGE, A. The plastic plant: root responses to heterogeneous supplies of nutrients. New Phytologist, v.162, p.9-24, 2004.

KING, J. S. et al. Belowground carbon input to soil is controlled by nutrient availability and fine root dynamics in loblolly pine. New

Phytologist, v.154, p.389-398, 2002.

LUY, A.; GOYA, J. F.; FRANGI, J. L. Distribución de la biomasa aérea y subterránea en plantaciones de Eucalyptus grandis de distintas edades en la región de Concordia, Entre Ríos (Argentina). In: CONGRESO FORESTALARGENTINO Y LATINO AMERICANO. Forestar y crecer. 12., 1997. PosadaMisiones: Asociación Forestal Argentina, 1997.

MAJDI, H. et al. Measuring fine root turnover in forest ecosystems. Plant and Soil, v.276, p.1-8, 2005.

MARSCHNER, H. Mineral nutrition of higher plants. 2.ed. San Diego: Academic Press, 1995. 889p.

MARTinS, L. G. C. Método de recomendação de adubação para eucalipto com base no monitoramento nutricional. 2004. 72f. Tese (Doutorado em Solos e Nutrição de Plantas) - Universidade Federal de Viçosa, Viçosa, MG, 2004.

Revista Árvore, Viçosa-MG, v.38, n.5, p.837-846, 2014 
MAURICE, J.et al. Fine root isotropy in Eucalyptus grandis plantations. Towards the prediction of root length densities from root counts on trench walls.

Plant and Soil, v.334, p.261-275, 2010.

MELLO, S. L. M.; GONÇALVES, J. L. M.; OLIVEIRA, L. E. G. Características do sistema radicular em povoamentos de eucaliptos propagados por sementes e estacas. Scientia Florestalis, v.54, p.16-26, 1998.

MOURA, O. N. et al. Conteúdo de nutrientes na parte aérea e eficiência nutricional em Mimosa caesalpiniifolia Benth. Revista Brasileira de Ciências Agrárias, v.1, n.1, p.23-29, 2006.

NEVES, J. C. L. Produção e partição de biomassa, aspectos nutricionais e hídricos em plantios clonais de eucalipto na região litorânea do Espírito Santo. 2000. 191f. Tese (Doutorado em Produção Vegetal) - Universidade Estadual do Norte Fluminense, Campos dos Goytacazes, 2000.

NORBY, R. J.; JACKSON, R. B. Root dynamics and global change: seeking an ecosystem perspective. New Phytologist, v.147, n.1, p.3$12,2000$.

OSTONEN, I. et al. Fine root morphological adaptations in Scots pine, Norway spruce and silver birch along a latitudinal gradient in boreal forests. Tree Physiology, v.27, p.1627-1634, 2007.

SCHUMACHER, M. V. Naehrstoffkreislauf in verschiedenen Bestaeden von Eucalyptus saligna Smith, Eucalyptus dunnii Maiden und Eucalyptus globules Labillardière in Rio Grande do Sul, Brasilien. 1995. 167f. Tese (Doutorado em
Ecologia e Nutrição Florestal) - Universitäet für Bodenkultur, Wien, 1995.

SCHUHLI, G. S.; PALUDZYSZYN FILHO, E. O cenário da silvicultura de teca e perspectivas para o melhoramento genético. Pesquisa Florestal Brasileira, v.30, n.63, p.217-230, 2010.

SCHWENGBER, D. R.; VENTURIN, N.; CARVALHO, J. G. Efeitos do gesso e calcário na relação raiz/parte aérea e em características das raízes de toças de seringueira (Hevea spp) recém implantadas em campo. Cerne, v.1, n.1, p.38-44, 1994.

STEENBJERG, F.; JAKOBSEN, S. T. Plant nutrition and yield curves. Soil Science, v.95, n.1, p.69-88, 1963.

TAIZ, L.; ZEIGER, E. Fisiologia vegetal. 3.ed. Porto Alegre: Artmed, 2004. 719p.

TSUKAMOTO FILHO, A. A. et al. Análise econômica de um plantio de teca submetido a desbastes. Revista Árvore, v.27, n.4, p.487-494, 2003.

VITOUSEK, P. M. Litterfall, nutrient cycling, and nutrient limitation in tropical forests. Ecology, v.65, n.1, p.285-298, 1984.

ZOBEL, R. W.; KINRAIDE, T. B.; BALIGAR, V. C. Fine root diameters can change in response to changes in nutrient concentrations. Plant and Soil, v.297, p.243-254, 2007.

ZHOU, Z. et al. Effects of calcium, boron and nitrogen fertilization on the growth of teak (Tectona grandis) seedlings and chemical property of acidic soil substrate. New Forests, v.42, n.2, p. (online first), 2011. 